\title{
Association between maternal acculturation and health beliefs related to oral health of Latino children
}

\author{
Tamanna Tiwari $^{1 *} \mathbb{D}$, Matthew Mulvahill ${ }^{2}$, Anne Wilson ${ }^{1}$, Nayanjot Rai ${ }^{3}$ and Judith Albino ${ }^{4}$
}

\begin{abstract}
Background: This report is presenting the association of maternal acculturation, measured by preferred language, and oral health-related psychosocial measures in an urban Latino population.

Methods: A cross-sectional survey was conducted with 100 mother-child dyads from the Dental Center at the Children's Hospital Colorado, the University of Colorado. A portion of Basic Research Factors Questionnaire capturing information about parental dental knowledge, attitudes, behavior and psychosocial measures was used to collect data from the participating mothers. Descriptive statistics were calculated for demographics and psychosocial measures by acculturation. A univariate linear regression model was performed for each measure by preferred language for primary analysis followed by adjusted model adjusting for parent's education.
\end{abstract}

Results: The mean age of the children was 3.99 years $(S D=1.11)$, and that of the mother was 29.54 years (SD =9.62). Dental caries, measured as dmfs, was significantly higher in children of Spanish-speaking mothers compared to children of English-speaking mothers. English-speaking mothers had higher mean scores of oral health knowledge, oral health behaviors, knowledge on dental utilization, self-efficacy, and Oral Health Locus of Control as compared to Spanish-speaking mothers. Univariate analysis demonstrated significant association for preference for Spanish language with knowledge on dental utilization, maternal self-efficacy, perceived susceptibility and perceived barriers. The effect of language was attenuated, but significant, for each of these variables after adjusting for parent's education.

Conclusion: This study reported that higher acculturation measured by a preference for the English language had a positive association with oral health outcomes in children. Spanish-speaking mothers perceived that their children were less susceptible to caries. Additionally, they perceived barriers in visiting the dentist for preventive visits.

Keywords: Oral health knowledge, Acculturation, Health belief model

\section{Background}

Acculturation is defined as the process by which individuals from a community may adopt the values and behaviors from another culture, and this may, in turn, affect their beliefs [1]. According to recent literature, acculturation is considered a multidimensional process, in which sociocultural changes may occur throughout the person's life course and varying even for individuals living within the same community [1-3].

\footnotetext{
* Correspondence: Tamanna.tiwari@ucdenver.edu

1Department of Community Dentistry and Population Health, School of Dental Medicine, University of Colorado Anschutz Medical Campus, Aurora, Colorado, USA

Full list of author information is available at the end of the article
}

There are many potential frameworks to understand the acculturation process and how it impacts health in immigrant populations. According to one theoretical framework, migration may cause psychosocial distress or loss of social support, resulting in a new behavioral environment that could be associated with the level of acculturation for an individual [3]. The framework presented by Fox et al. describes how socioeconomic conditions of migrant families may influence higher assimilation into the host culture, thereby resulting in higher levels of acculturation [3]. Furthermore, length of stay, language spoken at home, and social networks may influence the level of acculturation for an individual or a 
family $[4,5]$. For example, educational level, preferred language, and level of dental knowledge of the social networks are associated with a higher level of acculturation and, in turn, with the utilization of dental health services [6].

Another concept presented by Gao et al. discusses that the impact of acculturation on health can be mediated via a change in health behaviors and perceptions of one's ability to prevent disease or receive treatment [7]. In the context of health beliefs, acculturation can influence utilization of health services, doctor-patient communication, adopting new health behaviors, and treatment decisions $[7,8]$. Furthermore, acculturation may have a positive or negative effect on the health outcome in question [1]. For example, acculturation can be a risk factor for adoption of behaviors such as increased alcohol consumption, smoking, and higher fast food consumption, and on the other hand, it may promote increased amount of time engaged in exercise and physical activity [2].

For oral health outcomes, acculturation has been seen to influence the oral health behaviors, access, and navigation of oral healthcare [9, 10]. A recent systematic review of the impact of acculturation on oral health concluded that though higher levels of acculturation had a positive effect on utilization of dental services, this did not necessarily lead to improving oral health outcomes [7]. The authors also concluded that lower levels of acculturation might increase the risk of uptake of some unhealthy oral health-related behaviors, such as consumption of high sugary foods and drinks, and increase the risk of poor oral health outcomes [7]. Although there is a growing recognition that acculturation is a complicated process and that it influences oral health by a complex interplay of psychosocial factors, there is a scarcity of dental research addressing these issues.

Latinos are the largest and fastest immigrant group in the US, with a growth of $39 \%$ in Latino children between 2000 to 2010 [8]. In addition, within the $21 \%$ US households that speak a language other than English, $62 \%$ of these households are Spanish speaking [11]. Thus this study aimed to examine the association of maternal acculturation, measured as preferred language, and oral health-related psychosocial measures in an urban Latino population.

\section{Methods}

A cross-sectional survey was conducted with 100 mother-child dyads. One hundred dyads consisting of Latina mothers who were at least 18 years of age with a child under the age of 6 years were enrolled at the Dental Center at Children's Hospital Colorado, the University of Colorado in Aurora, Colorado. Latino families make up about $50 \%$ of the patient population at the Dental Center. The study only enrolled mothers who are primary caregivers to maintain reliability in the same kind of caregivers they study. A convenience sample of Latina mothers was enrolled from the waiting room of the Dental Center at Children's Hospital. A co-investigator on the study prescreened the mothers, and because Latina mothers were eligible for the study, pre-screening enabled appropriate enrollment. Information about the study was provided in English and Spanish, with a consent form detailing the approach of the study. Participating mothers were given the option to hear about the study details, sign the consent form and complete the survey in English or Spanish. Certified translators provided study information to Spanish-speaking mothers. This study was approved by the Colorado Multiple Institutional Review Board (COMIRB).

\section{Measures}

A manuscript with the main outcomes and study methodology has been recently published and presents all the details of the study design and implementation [12]. The questionnaire used in this study is a portion of the Basic Research Factors Questionnaire (BRFQ) [13] (Table 1), which captures parental dental knowledge, attitudes, behaviors, and other psychosocial measures. The BRFQ was developed as a collaborative effort involving three Centers for Research to Reduce Oral Health Disparities: located at the University of Colorado Denver, Boston University, and the University of California San Francisco (UCSF), and funded by the National Institute of Dental and Craniofacial Research. The BRFQ is available in English and Spanish and is being used by all three centers in diverse populations.

All the children participating in the study received an oral examination by one of the co-investigators of the study. Decayed, missing, filled, surfaces (dmfs) were measured during this examination. Data was recorded using an electronic dental research recording instrument designated as CARIN (Caries Research Instrument), specifically designed for research documentation involving dmfs.

Table 1 provides the description of the measures and the subscales of the BRFQ used in this study. Psychosocial measures include oral health behavior, oral health knowledge, knowledge on dental utilization, self-efficacy, three subscales on Oral Health Locus of Control, and four subscales from the Health Belief Model (Table 1). Knowledge on dental care utilization is another variable that measures the mother's knowledge about when to take the child for dental care, preventive visits vs. visiting the dentist in response to pain and the impact of her friends and family on her dental care utilization for her child. Behavior and oral health knowledge scales range from 0 to 100 and were calculated as the percent of individual items marked correctly. The remaining measures utilized Likert-type scales, with scores on each item ranging from 1 to 5 . The summary measures are the average of the values for answered questions, thus also ranging from 1 to 5 . 
Table 1 Description of Measures

\begin{tabular}{ll}
\hline Measure & Description \\
\hline $\begin{array}{l}\text { Oral Health Locus of Control } \\
\text { (OHLOC) } 3 \text { subscales }\end{array}$ & $\begin{array}{l}\text { OHLOC captures a person's attitudes about who or what has control over their child's oral health outcomes. } \\
\text { There are } 3 \text { subscales, which represents the extent to which participants believe control of their child's oral } \\
\text { health outcomes lies with the parent (internal LOC), the dentist (powerful other LOC), or chance factors } \\
\text { (chance LOC). }\end{array}$ \\
Health Belief Model 4 subscales & $\begin{array}{l}\text { The Health Belief Model is one of the major models that have been used to explain health behavior. The } \\
\text { model predicts that behavior is a function of } 4 \text { subscales - Perceived susceptibility, Perceived severity, } \\
\text { Perceived barriers, Perceived benefits. }\end{array}$ \\
Self-Efficacy & $\begin{array}{l}\text { Self-efficacy represents a person's confidence that he/she can successfully engage in a specific health } \\
\text { behavior. Self-efficacy score represents how sure participants are that they can engage in recommended } \\
\text { behavior to take care of their children's teeth. Ten questions quantify this measure. }\end{array}$ \\
Oral Health Behavior & $\begin{array}{l}\text { The overall behavior score represents the percentage of 13 oral health behavior items that were answered } \\
\text { with an adherent response, where "adherent" means the participant engages in the recommended oral health } \\
\text { behavior. } \\
\text { The overall knowledge score represents the percentage of (how many) oral health knowledge items answered } \\
\text { correctly. Nineteen questions quantify this measure. }\end{array}$ \\
Knowledge on dental utilization & Five items measured parental knowledge on utilization of oral health services for their children.
\end{tabular}

Language preference was used as a proxy for acculturation in this study. Several studies have used this proxy measure to capture acculturation, as use of non-native language has been associated with higher utilization of health services and better health in immigrant populations [5, 14]. Preference for English or Spanish was measured by asking the participating mothers if they wanted to complete the consent form and study survey in English or Spanish. Spanishspeaking mothers were considered to be less-acculturated

Table 2 Descriptive statistics for demographic variables by acculturation

\begin{tabular}{|c|c|c|}
\hline & language: English $(N=59)$ mean (SD) & language: Spanish $(N=40)$ mean $(S D)$ \\
\hline \multicolumn{3}{|l|}{ Gender } \\
\hline Male & $32(54.24 \%)$ & $21(52.50 \%)$ \\
\hline Female & $27(45.76 \%)$ & $19(47.50 \%)$ \\
\hline Child's Age & $3.94 \pm 1.09$ & $4.05 \pm 1.15$ \\
\hline Mother's Age & $28.20 \pm 9.77$ & $31.84 \pm 9.05$ \\
\hline \multicolumn{3}{|l|}{ Mother's Education } \\
\hline Less than HS & $17 / 56(30.36 \%)$ & 20/38 (52.63\%) \\
\hline HS or more & $39 / 56(69.64 \%)$ & 18/38 (47.37\%) \\
\hline \multicolumn{3}{|l|}{ Mother's Employment } \\
\hline Employed & $23(38.98 \%)$ & $12(30.00 \%)$ \\
\hline Not employed & $36(61.02 \%)$ & $28(70.00 \%)$ \\
\hline Household Size & $4.66 \pm 1.53$ & $4.89 \pm 1.23$ \\
\hline \multicolumn{3}{|l|}{ Household Income } \\
\hline$\$ 10,830-\$ 14,569$ & $3 / 28(10.71 \%)$ & $1 / 13(7.69 \%)$ \\
\hline$\$ 14,570-\$ 18,309$ & $3 / 28(10.71 \%)$ & $1 / 13(7.69 \%)$ \\
\hline$\$ 18,310-\$ 22,049$ & $2 / 28(7.14 \%)$ & $3 / 13(23.08 \%)$ \\
\hline$\$ 22,050-\$ 25,789$ & $3 / 28(10.71 \%)$ & $2 / 13(15.38 \%)$ \\
\hline$\$ 25,790-\$ 29,529$ & $12 / 28(42.86 \%)$ & $3 / 13(23.08 \%)$ \\
\hline$\$ 29,530-\$ 33,269$ & 0/28 (0.00\%) & 2/13 (15.38\%) \\
\hline$\$ 33,270-\$ 37,009$ & $3 / 28(10.71 \%)$ & $1 / 13(7.69 \%)$ \\
\hline$\$ 37,010$ & $2 / 28(7.14 \%)$ & 0/13 (0.00\%) \\
\hline Household Minors & $2.61 \pm 1.42$ & $2.72 \pm 1.28$ \\
\hline Household Years in Household & $4.58 \pm 3.67$ & $3.79 \pm 3.25$ \\
\hline Dental Caries (dmfs) & $7.56 \pm 12.11$ & $15.20 \pm 21.48$ \\
\hline
\end{tabular}


or closer to the Latino culture, as compared to English-speaking.

\section{Data analysis}

Analyses for this study tested the effect of preferred language, which was considered a proxy for acculturation, on psychosocial measures of oral health. Descriptive statistics were calculated for demographics and psychosocial measures by acculturation. Descriptive statistics presented for continuous variables are both mean (SD) and median (IQR), where IQR is the $25 \%$ and $75 \%$ or inter-quartile interval. For categorical variables, count and percentages are presented. The primary analysis consists of univariate linear regression models for each measure by preferred language, as well as a model adjusting for parent's education. A significance level of 0.05 was used in all hypothesis testing and confidence intervals. Data cleaning and analysis were conducted using R version 3.3.2 (2016-10-31) (R Core Team 2013).

\section{Results}

Table 2 provides demographic characteristics of the study sample, presently separately by the preferred language of communication. Fifty-nine mothers were English speaking, and 40 were a Spanish speaker. The mean age of the children in the study was 3.99 years $(\mathrm{SD}=1$. $11)$, and that of the mother was 29.54 years $(S D=9.62)$. Dental caries, measured as dmfs, was significantly higher $(15.20 \pm 21.48)$ in children of Spanish-speaking mothers than for children of English-speaking mothers $(7.56 \pm 12$. 11) $(p=0.043)$.

Table 3 provides the means of the psychosocial measures by preferred language. English-speaking mothers had higher mean scores of oral health knowledge (87.51 $\pm 7.65)$, behaviors $(47.13 \pm 14.98)$, knowledge of dental utilization (3.67 \pm 0.51$)$, and self-efficacy $(4.34 \pm 0.59)$, compared to Spanish-speaking mothers. Englishspeaking mothers had higher scores of Internal Oral Health Locus of Control (4.38 \pm 0.68$)$; Spanish-speaking mothers scored higher on one subscale of the Health Belief Model, Perceived Barriers (4.07 \pm 1.25$)$.

In the univariate models (Table 4), the preference for Spanish-language was significantly associated with knowledge of dental utilization $(-0.51, P=0.0006)$, maternal self-efficacy $(-0.44, P=0.0024)$. Preference for the Spanish language was also significantly associated with maternal Perceived Susceptibility $(-0.52, P$ $=0.0046)$ Perceived Barriers $(0.53, P=0.0001)$, and two subscales of Health Belief Model. After adjusting for parent's education, the effect of language was slightly attenuated, though still significant, for each of these variables, suggesting that parent's education was a significant confounder.
Table 3 Descriptive statistics for psychosocial measures by acculturation

\begin{tabular}{|c|c|c|}
\hline & language: English $(N=59)$ & language: Spanish $(N=40)$ \\
\hline \multicolumn{3}{|c|}{ Oral health Behavior $(P=0.0988)$} \\
\hline mean (SD) & $47.13 \pm 14.98$ & $41.76 \pm 16.18$ \\
\hline median (IQR) & $50.00(34.85,58.33)$ & $41.67(31.82,50.00)$ \\
\hline \multicolumn{3}{|c|}{ Oral health knowledge $(P=0.3995)$} \\
\hline mean (SD) & $87.51 \pm 7.65$ & $85.67 \pm 12.18$ \\
\hline median (IQR) & $88.89(82.35,93.54)$ & $88.24(82.35,94.12)$ \\
\hline \multicolumn{3}{|c|}{ Knowledge on dental utilization $(P=0.0024)$} \\
\hline mean (SD) & $3.67 \pm 0.51$ & $3.15 \pm 0.93$ \\
\hline median (IQR) & $3.60(3.40,4.00)$ & $3.40(2.45,4.00)$ \\
\hline \multicolumn{3}{|c|}{ Self-efficacy $(P=0.0043)$} \\
\hline mean (SD) & $4.34 \pm 0.59$ & $3.91 \pm 0.79$ \\
\hline median (IQR) & $4.50(4.00,4.80)$ & $4.06(3.60,4.45)$ \\
\hline \multicolumn{3}{|c|}{ Locus of control - Internal $(P=0.1655)$} \\
\hline mean (SD) & $4.38 \pm 0.68$ & $4.07 \pm 1.25$ \\
\hline median (IQR) & $4.67(4.00,5.00)$ & $4.67(3.67,5.00)$ \\
\hline \multicolumn{3}{|c|}{ Locus of control - External Others $(P=0.6971)$} \\
\hline mean (SD) & $2.19 \pm 1.01$ & $2.27 \pm 0.94$ \\
\hline median (IQR) & $2.00(1.33,2.67)$ & $2.33(1.67,3.00)$ \\
\hline \multicolumn{3}{|c|}{ Locus of control - External Chance $(P=0.6227)$} \\
\hline mean (SD) & $2.10 \pm 1.01$ & $2.22 \pm 1.19$ \\
\hline median (IQR) & $2.00(1.33,3.00)$ & $2.00(1.00,3.00)$ \\
\hline \multicolumn{3}{|c|}{ Health belief model - Severity $(P=0.0574)$} \\
\hline mean (SD) & $4.29 \pm 0.94$ & $3.94 \pm 0.82$ \\
\hline median (IQR) & $4.67(3.67,5.00)$ & $3.67(3.58,5.00)$ \\
\hline \multicolumn{3}{|c|}{ Health belief model - Barriers $(P=0.0002)$} \\
\hline mean (SD) & $1.89 \pm 0.61$ & $2.42 \pm 0.68$ \\
\hline median (IQR) & $1.80(1.45,2.20)$ & $2.45(1.80,3.00)$ \\
\hline \multicolumn{3}{|c|}{ Health belief model - Susceptibility $(P=0.0080)$} \\
\hline mean (SD) & $3.34 \pm 0.75$ & $2.83 \pm 1.03$ \\
\hline median (IQR) & $3.50(3.00,3.75)$ & $3.00(2.00,3.50)$ \\
\hline \multicolumn{3}{|c|}{ Health belief model - Benefits $(P=0.1951)$} \\
\hline mean (SD) & $4.31 \pm 0.67$ & $4.03 \pm 1.24$ \\
\hline median (IQR) & $4.40(4.20,4.80)$ & $4.55(3.70,5.00)$ \\
\hline
\end{tabular}

\section{Discussion}

The impetus for acculturation research in health is to determine whether experiences of a particular group have effects on trends in health outcome [15]. In this study, higher acculturation, which was measured by a preference for the English language had a positive association with oral health outcomes, i.e., dental caries in children. Children of English-speaking mothers had significantly lower dmfs scores compared with children of Spanish-speaking mothers. Spanish-speaking mothers had low knowledge of dental care utilization, low self- 
Table 4 Linear regression of each psychosocial variable on primary language (Spanish) on both a univariate basis and adjusted for parent's education

\begin{tabular}{lllll}
\hline Outcome & estimate $^{*}$ & $P$. value & Edu Adj. estimate $^{*}$ & Edu Adj. $p$.value $^{*}$ \\
\hline Oral health Behavior & $-5.37(-11.66,0.92)$ & $P=0.0932$ & $-5.69(-12.21,0.84)$ & $P=0.0867$ \\
Oral health Knowledge & $-1.84(-5.79,2.11)$ & $P=0.3581$ & $-1.41(-5.45,2.63)$ & $P=0.4894$ \\
Knowledge on dental service utilization & $-0.51(-0.80,-0.22)$ & $P=0.0006$ & $-0.41(-0.71,-0.11)$ & $P=0.0073$ \\
Self-efficacy & $-0.44(-0.71,-0.16)$ & $P=0.0024$ & $-0.35(-0.62,-0.09)$ & $P=0.0099$ \\
HBM - Severity & $-0.34(-0.71,0.02)$ & $P=0.0636$ & $-0.26(-0.65,0.12)$ & $P=0.1800$ \\
HBM - Barriers & $0.53(0.27,0.79)$ & $P=0.0001$ & $0.46(0.20,0.71)$ & $P=0.0006$ \\
HBM - Susceptibility & $-0.52(-0.87,-0.16)$ & $P=0.0046$ & $-0.41(-0.78,-0.05)$ & $P=0.0273$ \\
HBM - Benefits & $-0.28(-0.66,0.10)$ & $P=0.1477$ & $-0.20(-0.57,0.18)$ & $P=0.3045$ \\
LOC - Internal & $-0.30(-0.69,0.08)$ & $P=0.1213$ & $-0.20(-0.57,0.17)$ & $P=0.2867$ \\
LOC - External Others & $0.08(-0.32,0.48)$ & $P=0.7012$ & $0.07(-0.36,0.49)$ & $P=0.7600$ \\
LOC - External Chance & $0.11(-0.33,0.56)$ & $P=0.6114$ & $0.10(-0.38,0.58)$ & $P=0.6715$ \\
\hline
\end{tabular}

*Estimates are the difference in the psychosocial measure from primarily English-speaking and Spanish-speaking patients

efficacy; they perceived their children not susceptible to dental caries and perceived more barriers in visiting the dentist for prevention visits.

Our assumption was that recruiting a health center population should have neutralized any potential structural barriers related to utilization or access to care. However, Spanish-speaking mothers in this study perceived barriers in visiting the dentist for preventive visits and had poor oral health behaviors towards their children, when compared with English-speaking mothers. They also had perceptions that their children are less susceptible to developing dental caries, an attitude that may suggest why they did not do much about preventing dental caries. Spanish-speaking mothers had less knowledge related to dental care utilization, including timing and necessity of a dental visit, and they submitted to the notion that young children should only be taken for a dental visit if they were in pain. Furthermore, their social networks, including family and friends, supported not visiting the dentist unless there was an acute need, and they agreed that no one in their family told them the child should be taken for a preventive dental visit.

These perceptions could be related to cultural norms and beliefs, and because Spanish-speaking mothers were less acculturated compared with English-speaking mothers, their beliefs related to preventive dental visits would be inclined towards the Latino cultural norms [8], which in one study that was conducted with Mexican Americans has been described as a "reactive orientation" towards dental care [4]. Previous research also has shown that Latino parents can have fatalistic attitudes towards the oral health of their children and reduced utilization due to perceived barriers [16, 17]. Another study that collected qualitative data from Spanishspeaking Latina mothers at the same health center reported that they struggled to overcome the familial pressure against visiting the dentist for preventive appointments for their children and received suggestions that dental visits should be done in response to pain [18].

These differences in maternal oral health beliefs and behaviors can be embedded theoretically in the level of assimilation into the Anglo culture [2]. For example, partial acculturation of Latino families- that is, alienation from their traditional culture and incomplete integration into the new culture, may put them at greater risk for poor oral health related behaviors, which may also impact utilization and ultimately disease outcome [7, 8].

Although acculturation was measured by the proxy variable of preferred language use, it still shows an influence on maternal beliefs and perceptions related to caries prevention and dental care utilization - and ultimately the dental caries experience in children in this study. Lacking the ability to speak English can be seen as an external manifestation of "slow cultural transformation," which may influence access to care and navigation of the healthcare system, thus indirectly affecting health outcomes [19]. Within pediatric oral health research, lower dental care utilization, lower sealant applications and delayed first dental visits have been related to non-English speaking households [8]. For nonEnglish speaking mothers, it may become difficult to follow the oral hygiene instructions and recommended behaviors given at the dental office, and because of the language barrier, their social networks might be limited to Spanish-speaking individuals. Recent studies have shown the complex role of social networks and acculturation in oral health [4]. Moreover, it has been reported that reduced English skills may cause significant difficulties for Latino families, making them less trusting towards dental care professionals and not establishing a dental home [10, 20]. 
The present study has some limitations. We drew our sample from a conveniently available urban health center population that may not be generalizable to the larger population. Acculturation was measured using the proxy variable of language preference, and we did not collect information on the birthplace of the mother if she was U.S. born or foreign born.

\section{Conclusion}

This study makes important contributions to understanding the underlying psychosocial and behavioral factors that predict how acculturation impacts oral health. Further longitudinal research is warranted in this direction, in using a multi-dimensional tool to measure acculturation rather than using a proxy variable. The author has secured funding to conduct a study that will use a multidimensional scale to measure acculturation.

\section{Abbreviations}

BRFQ: Basic Research Factors Questionnaire; IQR: Interquartile Range;

SD: Standard Deviation; UCSF: University of California San Francisco

\section{Acknowledgements}

We would like to thank Dr. Sarah Horton and Dr. Sarah R. Baker for their comments on the draft. We would especially like to thank the children and their families who have participated in the study.

\section{Funding}

The grant support for this project is National Institute of Dental and Craniofacial Research award number K99DE024758 and R00DE024758.

\section{Availability of data and materials}

Data will be made available based on the NIH Data Sharing Policy. The datasets used and/or analyzed during the current study are available from the corresponding author on reasonable request.

\section{Authors' contributions}

TT designed the study, acquisition of the data, contributed to data analysis, data interpretation, manuscript writing and final approval of the manuscript. MM contributed to the analysis, interpretation, critically revised the manuscript and provided final approval of the manuscript. AW and JA contributed to the conception, design, critically revised the manuscript and provided final approval of the manuscript. NR contributed to the manuscript writing, acquisition of data and management of data, critically revised the manuscript and provided final approval of the manuscript. All authors read and approved the final manuscript. All authors agree to be accountable for all aspects of the work.

\section{Ethics approval and consent to participate}

The study was approved by the University of Colorado IRB - Colorado Multiple Institutional Review Board (COMIRB) and all study participants signed a consent form before starting the study. Consent was obtained from parents/legal guardians before any data was collected form minors and children

\section{Competing interests}

The authors declare that they have no competing interests with respect to the authorship and/or publication of this article.

\section{Publisher's Note}

Springer Nature remains neutral with regard to jurisdictional claims in published maps and institutional affiliations.

\section{Author details}

'Department of Community Dentistry and Population Health, School of Dental Medicine, University of Colorado Anschutz Medical Campus, Aurora, Colorado, USA. ${ }^{2}$ School of Medicine, University of Colorado Anschutz Medical Campus, Aurora, Colorado, USA. ${ }^{3}$ Colorado School of Public Health, University of Colorado Anschutz Medical Campus, Aurora, Colorado, USA. ${ }^{4}$ Center for Native Oral Health Research, University of Colorado Anschutz Medical Campus, Aurora, Colorado, USA.

Received: 22 May 2017 Accepted: 17 April 2018

Published online: 24 April 2018

\section{References}

1. Abraído-Lanza AF, Armbrister AN, Flórez KR, Aguirre AN. Toward a theorydriven model of acculturation in public Health Research. Am J Public Health. 2006;96:1342-6. https://doi.org/10.2105/AJPH.2005.064980.

2. Abraído-Lanza AF. Social support and psychological adjustment among Latinas with arthritis: a test of a theoretical model. Ann Behav Med. 2004;27: 162-71. https://doi.org/10.1207/s15324796abm2703_4.

3. Fox M, Entringer S, Buss C, DeHaene J, Wadhwa PD. Intergenerational transmission of the effects of acculturation on health in Hispanic Americans: a fetal programming perspective. Am J Public Health. 2015;105(Suppl 3): S409-23. https://doi.org/10.2105/AJPH.2015.302571.

4. Maupome G, McConnell WR, Perry BL, Marino R, Wright ER. Psychological and behavioral acculturation in a social network of Mexican Americans in the United States and use of dental services. Community Dent Oral Epidemiol. 2016;44:540-8. https://doi.org/10.1111/cdoe.12247.

5. Hunt LM, Schneider S, Comer B. Should "acculturation" be a variable in health research? A critical review of research on US Hispanics. Soc Sci Med. 2004;59:973-86. https://doi.org/10.1016/j.socscimed.2003.12.009.

6. Pullen E, Perry BL, Maupome G. "Does this look infected to you?" social network predictors of dental help-seeking among Mexican immigrants. J Immigr Minor Health. 2017:1-11. https://doi.org/10.1007/s10903-017-0572-x.

7. Gao X-L, McGrath C. A review on the oral health impacts of acculturation. J Immigr Minor Health. 2011;13:202-13. https://doi.org/10.1007/s10903-0109414-9.

8. Tiwari T, Albino J. Acculturation and pediatric minority oral health interventions. Dental Clinics. 2017;61(95):35-42.

9. Farokhi MR, Cano SM, Bober-Moken IG, Bartoloni JA, Cunningham SE, Baez MX. Maternal acculturation could it impact oral health practices of MexicanAmerican mothers and their children? J Prim Care Community Health. 2011; 2:87-95. https://doi.org/10.1177/2150131910388942.

10. Patrick DL, Lee RSY, Nucci M, Grembowski D, Jolles CZ, Milgrom P. Reducing oral health disparities: a focus on social and cultural determinants. BMC Oral Health. 2006;6(Suppl 1):S4. https://doi.org/10.1186/1472-6831-6-S1-S4.

11. Zong J, Batalova J. Frequently requested statistics on immigrants and immigration in the United States. Washington, DC: Migration Policy Institute; 2016. http://www.migrationpolicy.org/article/frequentlyrequestedstatistics-immigrants-and-immigration-united-states\#Top. Assessed Aug 242016.

12. Tiwari T, Wilson AR, Mulvahill M, Rai N, Albino J. Maternal Factors Associated with Early Childhood Caries in Urban Latino Children. JDR Clin Trans Res. 2017; https://doi.org/10.1177/2380084417718175.

13. Albino J, Tiwari T, Gansky SA, Barker JC, Brega AG, Gregorich S, et al. The basic research factors questionnaire for studying early childhood caries. BMC Oral Health. 2017;17:83. https://doi.org/10.1186/s12903-017-0374-5.

14. Abraído-Lanza AF, Chao MT, Florez KR. Do healthy behaviors decline with greater acculturation?: Implications for the Latino mortality paradox. Soc Sci Med. 2005:61(6):1243-55.

15. Fox M, Thayer Z, Wadhwa PD. Assessment of acculturation in minority health research. Soc Sci Med. 2017;176:123-32. https://doi.org/10.1016/j. socscimed.

16. Butani Y, Weintraub JA, Barker JC. Oral health-related cultural beliefs for four racial/ethnic groups: assessment of the literature. BMC Oral Health. 2008;8:26. https://doi.org/10.1186/1472-6831-8-26

17. Hoeft KS, Barker JC, Masterson EE. Urban Mexican-American mothers' beliefs about caries etiology in children. Community Dent Oral Epidemiol. 2010;38: 244-55. https://doi.org/10.1111/j.1600-0528.2009.00528.x.

18. Tiwari T, Sharma T, Gutierrez K, Wilson A, Albino J. Learning about Oral Health Knowledge \& Behavior in Latina Mothers. Boston: IADR/AADR/CADR General Session \& Exhibition; 2015. 
19. Flores G, Tomany-Korman SC. Racial and ethnic disparities in medical and dental health, access to care, and use of services in US children. Pediatrics. 2008;121:e286-98.

20. Graham MA, Tomar SL, Logan HL. Perceived social status, language and identified dental home among Hispanics in Florida. J Am Dent Assoc. 2005; 136:1572-82.

Ready to submit your research? Choose BMC and benefit from:

- fast, convenient online submission

- thorough peer review by experienced researchers in your field

- rapid publication on acceptance

- support for research data, including large and complex data types

- gold Open Access which fosters wider collaboration and increased citations

- maximum visibility for your research: over $100 \mathrm{M}$ website views per year 